\title{
Jean Meynaud
}

Professeur de science politique

Aux Universités de Genève et de Lausanne

(1972)

\section{La dictature grecque a cinq ans}

\author{
Un document produit en version numérique par Mme Marcelle Bergeron, bénévole \\ Professeure à la retraite de l'École Dominique-Racine de Chicoutimi, Québec \\ et collaboratrice bénévole \\ Courriel : Marcelle_Bergeron@uqac.ca \\ Dans le cadre de la collection : "Les classiques des sciences sociales" \\ dirigée et fondée par Jean-Marie Tremblay, \\ professeur de sociologie au Cégep de Chicoutimi \\ Site web: http://classiques.uqac.ca/ \\ Une collection développée en collaboration avec la Bibliothèque \\ Paul-Émile-Boulet de l'Université du Québec à Chicoutimi \\ Site web:_ http://bibliotheque.uqac.ca/
}




\section{Politique d'utilisation de la bibliothèque des Classiques}

Toute reproduction et rediffusion de nos fichiers est interdite, même avec la mention de leur provenance, sans l'autorisation formelle, écrite, du fondateur des Classiques des sciences sociales, Jean-Marie Tremblay, sociologue.

Les fichiers des Classiques des sciences sociales ne peuvent sans autorisation formelle:

- être hébergés (en fichier ou page web, en totalité ou en partie) sur un serveur autre que celui des Classiques.

- servir de base de travail à un autre fichier modifié ensuite par tout autre moyen (couleur, police, mise en page, extraits, support, etc...),

Les fichiers (.html, .doc, .pdf, .rtf, .jpg, .gif) disponibles sur le site Les Classiques des sciences sociales sont la propriété des Classiques des sciences sociales, un organisme à but non lucratif composé exclusivement de bénévoles.

Ils sont disponibles pour une utilisation intellectuelle et personnelle et, en aucun cas, commerciale. Toute utilisation à des fins commerciales des fichiers sur ce site est strictement interdite et toute rediffusion est également strictement interdite.

\section{L'accès à notre travail est libre et gratuit à tous les utilisateurs. C'est notre mission.}

Jean-Marie Tremblay, sociologue

Fondateur et Président-directeur général, LES CLASSIQUES DES SCIENCES SOCIALES. 
Un document produit en version numérique par Mme Marcelle Bergeron, bénévole, professeure à la retraite de l’École Dominique-Racine de Chicoutimi, Québec.

Courriel : marcelle_bergeron@uqac.ca

Jean MEYNAUD

La dictature grecque a cinq ans. Montréal : Les Éditions Nouvelle Frontière, 1972, 45 p.

[Autorisation formelle accordée parles ayant-droit de l'œuvre de Jean Meynaud, son épouse, Mme Meynaud-Zogrphos, Michel Meynaud (fils) et Hélène-Yvonne Meynaud (fille) le 19 octobre 2008 de diffuser toutes les œuvres de Jean Meynaud dans Les Classiques des sciences sociales.]

Bf Courriels :

Mme Meynaud-Zographos (épouse) : meynaud-zographos@orange.fr

Hélène-Yvonne Meynaud (fille) : $\quad$ hymeynaud@free.fr.

Polices de caractères utilisés :

Pour le texte : Times New Roman, 12 points.

Pour les citations : Times New Roman 10 points.

Pour les notes de bas de page : Times New Roman, 10 points.

Mise en page sur papier format : LETTRE (US letter), 8.5’’ x 11'’)

Édition électronique réalisée avec le traitement de textes Microsoft Word 2007 pour Windows.

Édition réalisée le 22 juin 2010 et complétée le 28 juillet 2010 à Chicoutimi, Ville de Saguenay, Québec. 


\section{Jean Meynaud}

(1972)

La dictature grecque a cinq ans.

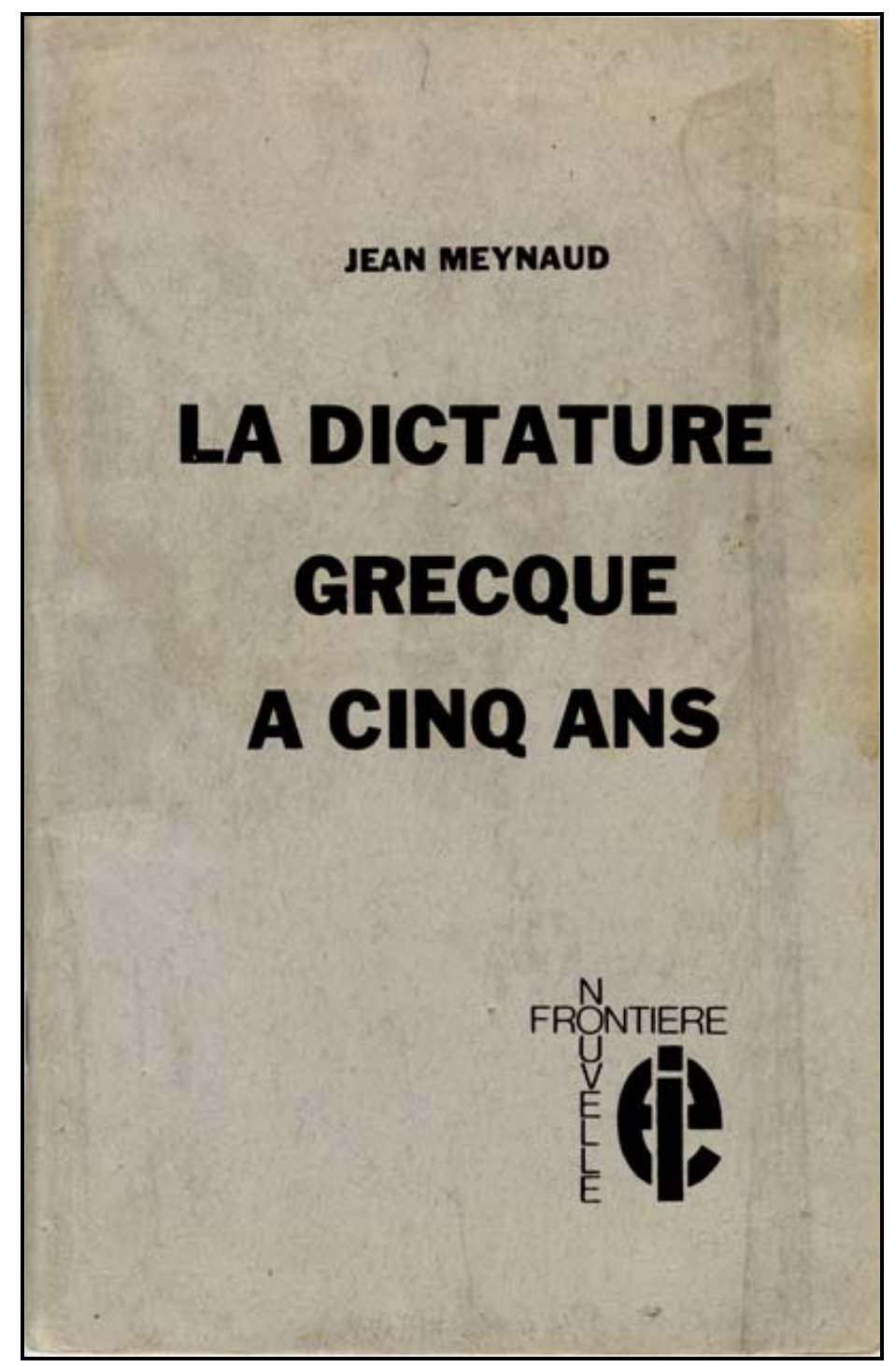

Montréal : Les Éditions Nouvelle Frontière, 1972, 45 p. 


\section{Table des matières}

$\underline{\text { Introduction }}$

I. Signification générale de la dictature

II. Portée de l'affrontement entre la Junte et la Droite

III. À propos des tactiques unitaires

IV. Au peuple grec de choisir sa destinée. 
[p. 5]

«La dictature grecque a cinq ans » n'était pas, au départ, un manuscrit destiné à la publication. Préparé pour être distribué aux participants du Congrès International pour l'Abolition de la Dictature en Grèce (Paris les 17, 18 et 19 mars 1972), il ne devait servir qu'à faire connaître au Congrès les positions de son auteur et procurer une base à une discussion fructueuse.

Le décès de Jean Meynaud, le lendemain du jour où ce brouillon était terminé, a décidé l'équipe Meynaud à publier ce texte, exactement tel qu'il a été trouvé dans la serviette de travail.

Ce fut toujours le vœu du Patron que son équipe ouvre, dans le cadre d'une libération de tous les peuples opprimés, à la libération de la Grèce.

[p. 6, sans texte] 
[p. 7]

\section{INTRODUCTION}

$\underline{\text { Retour à la table des matières }}$

L'objet de ce court essai n'est pas de dresser un bilan des structures et des activités de la dictature qui écrase la Grèce depuis cinq ans. Un tableau de cet ordre même réduit à l'essentiel, exigerait pour avoir quelque signification un cadre bien plus vaste. Il s'agit seulement, en ces pages, de dégager un fil directeur qui permette d'analyser correctement les intentions et les démarches des putschistes comme aussi de comprendre, du même coup, les mobiles des différents groupes d'opposition.

La tyrannie, instaurée le 21 avril 1967 et maintenue depuis sans modification substantielle, n'est pas un accident. Elle représente simplement un aspect, particulièrement sauvage et répugnant, des efforts entrepris, avec beaucoup de constance, pour bloquer l'évolution sociale et politique de la Grèce, pour empêcher les transformations économiques de retentir sur la distribution du pouvoir. De Metaxás à Papadopoulos, en passant par Papagos et Caramanlis, il n'y a pas de solution de continuité. Or le régime militaire qui, comme ses prédécesseurs de la Droite, joue à fond la carte des possédants, s'est trouvé en proie, presque dès le début, aux critiques et dénonciations de porte-parole de ces milieux. Corollaire de ces attaques il est vrai : la propagande pour des formules de remplacement dont l'inspiration fondamentale consiste dans la remise en vigueur des procédés anciens de la sauvegarde bourgeoise. Si ces projets aboutissaient, la dictature n'aurait été qu'une parenthèse, ouverte et fer[p. 8] mée sans que les classes privilégiées dans leur ensemble aient cessé de tirer des avantages matériels de la situation.

Il s'agit donc, en premier lieu, de bien comprendre les mobiles des assauts venus de la Droite. Mais les formules proposées par celle-ci exercent une influence au-delà même des milieux ayant pour seul critère la conservation intégrale du système social établi. Ainsi des groupes d'inspiration libérale, voire progressiste, participent-ils, pour des raisons diverses à une œuvre de mystification qui tend, de manière pourtant évidente, à brouiller les responsabilités et à assurer la perpétuation d'un système dont le putsch d'avril 1967 a été le fruit naturel. Il est dès lors nécessaire de spécifier les fondements et les risques de cette mystification - cet effort de classification 
impliquant, on doit l'admettre dès le début, une évaluation critique des appels à l'union nationale dans l'état actuel du rapport des forces. La notion d'un regroupement de tous ceux qui, pour une raison ou l'autre, s'opposent à la Junte n'est pas seulement un mirage dont la poursuite serait susceptible de faire perdre inutilement beaucoup de temps et d'énergie : elle constitue une faute politique de première grandeur et ne peut avoir objectivement d'autre résultat qu'un nouveau renforcement de la Droite. 
[p. 9]

\section{I \\ SIGNIFICATION GÉNÉRALE DE LA DICTATURE}

$\underline{\text { Retour à la table des matières }}$

À première vue, il paraît difficile de trouver un principe systématique d'interprétation convenant à un régime qui allie la tyrannie avec le désordre sans que le second de ces traits tempère nécessairement le premier. Pourtant de multiples aspects de ce sinistre dossier s'éclairent si l'on observe que le régime des colonels utilise des moyens dictatoriaux pour finalement mener la plus banale des politiques capitalistes, qu'il emploie tout un arsenal de procédés tyranniques pour défendre un ordre social, dont, vu la situation des forces après la guerre civile, la répression policière habituelle suffisait amplement à assurer la sauvegarde.

Depuis qu'ils se sont emparés de l'appareil gouvernemental, les militaires factieux se bornent à faire la politique de la Droite avec d'autres moyens - cette politique dont le Centre s'était appliqué, durant son passage au gouvernement à modifier certaines des orientations, de manière d'ailleurs relative et en évitant de brusquer, autrement que de manière verbale, la classe des possédants. Il suffira pour établir cette continuité de mention-[p. 10] ner quelques têtes de rubrique : introduction en Grèce de la société de consommation avec ses tares, ses injustices et ses distorsions habituelles; constitution du pays en réserve pour le capital monopoliste étranger et assujettissement de l'économie aux impulsions venues de centres de décision extérieurs; subordination de la croissance nationale à l'essor des grands pays capitalistes, le développement grec n'étant ainsi qu'un sous-produit du capitalisme mondial; érection des armateurs, ces champions de la fraude fiscale et ces spécialistes de la désertion nationale, en caste dotée de privilèges exorbitants ; domestication des syndicats ouvriers par les soins conjugués du patronat et de la police ; recours massif à l'émigration tant comme soupape de sûreté socio-politique que comme mode de financement des comptes extérieurs; gonflement ininterrompu de la dette contractée auprès des prêteurs étrangers ; fidélité exemplaire à l'alliance atlantique et propension notoire à rechercher aux dépens de l'hellénisme une solution à la crise chypriote qui permette d'intégrer l'île dans le dispositif de l'OTAN. 
Il ne serait pas difficile d'allonger cette liste de convergences profondes. Soit, par exemple, le secteur-clé de l'éducation. L'opposition a vigoureusement protesté contre le démantèlement des réformes introduites par G. Papandréou sous le gouvernement de l'Union du Centre et des voix de la Droite se sont jointes à ce concert de dénonciations. L'on ne saurait oublier pour autant les campagnes farouches menées contre l'adoption de ces réformes par les journalistes de la Droite, certains de ceux-ci n'hésitant pas à les dénoncer comme un nouvel élément de la soi-disant conspiration communiste. Sur ce point, comme sur tant d'autres, les colonels se sont bornés à épouser les positions les plus obscurantistes d'une Droite encore fort éloignée du monde moderne.

En définitive, la Junte n'a pas dévié de la route tracée ou préconisée par la Droite : comme cette dernière, quoi-[p. 11] qu'avec un style différent, elle a continué d'engager le pays sans cesse plus avant sur la voie de la servitude économique et militaire. Quiconque a étudié en leur temps les activités gouvernementales de l'ERE n'éprouve aucun sentiment de nouveauté en observant celles de la Junte. Le colonel Papadopoulos, partisan, selon ses propres déclarations, de C. Caramanlis à l'époque du régime parlementaire, n'a certes rien oublié, rien renié de ses préférences anciennes.

Les maîtres actuels de la Grèce ne s'en tiennent pas, il est vrai, aux techniques de la démocratie policière en honneur à l'époque caramanlique. La dictature a utilisé, d'une manière ou d'une autre, souvent de façon féroce, tous les procédés du totalitarisme moderne : déportation et camp de concentration, torture, chantage et délation à très grande échelle, contrôle absolu des moyens de communication, surveillance étroite des agents publics et parapublics, épuration constante des administrations civiles et des corps militaires, embrigadement de l'Église et destruction méthodique de la liberté d'expression sous toutes ses formes, suppression des franchises universitaires, intimidation psychologique, retrait aux adversaires de toute possibilité de travail (y compris dans le secteur privé de l'économie), essai d'endoctrinement de la jeunesse...

Aujourd'hui certains de ces moyens font l'objet d'une utilisation moins intense ou moins large dans le but évident de désarmer les critiques faites à l'étranger. C'est le processus dit de «libéralisation" du régime, lequel donne lieu à toute une propagande qui n'impressionne pas seulement les touristes distraits et les sympathisants idéologiques. En réalité, les putschistes ont appris à mieux se servir de l'arme de la peur. Dans un pays entièrement quadrillé par des forces de police qui utilisent des hordes de mouchards, l'efficacité de la répression n'exige pas que l'on garde en prison des milliers de gens mais que tout citoyen puisse, le cas échéant sur la base [p. 12] de la dénonciation la plus vile, être privé de sa liberté De la même manière, la menace de brutalité ou de torture n'exige pas pour être efficace que ces procédés soient appliqués à des masses de gens : il suffit de savoir que toute personne arrêtée pour faits de résistance ou simplement soupçonnée à ce titre risque de subir de tels traitements. À leur niveau actuel, les détentions, arrestations, persécutions et procès suffisent amplement pour que l'atmosphère du régime demeure, comme elle a toujours été, d'inspiration nettement terroriste. Le pays tout entier baigne dans un 
climat de surveillance et d'oppression policières. Et le régime ne saurait renoncer à cette protection sans mettre en marche du même coup l'engrenage de sa propre destruction. En définitive, ce sont toujours les procédés employés pour mater les opposants, contrôler les suspects et décourager les protestataires éventuels qui constituent la principale originalité du gouvernement, la seule même, si du moins l'on néglige certaines nuances secondaires, par rapport à ses devanciers de la Droite.

Certes même sous l'angle de la répression, la conduite de cette dernière a comporté des pages fort noires et qu'il n'est pas légitime d'expliquer toutes par les désastres de la guerre civile. L'Union du Centre elle-même n'a finalement pas ou le courage d'abolir dans son intégralité ce dispositif dit d'exception mais qui, depuis Metaxás, a formé un trait permanent de la vie publique en Grèce. Ainsi les colonels peuvent-ils se servir pour persécuter leurs compatriotes de textes largement antérieurs au putsch et dont les gouvernements précédents n'avaient pas voulu ou pas osé entreprendre l'abrogation. Et, s'ils ont besoin d'arguments contre le « communisme », c'est-à-dire contre toute pensée politique humaniste ou progressiste, les journaux publiés par Hélène Vlachos au temps du régime constitutionnel apportent à cet égard une mine inépuisable.

Il serait donc très excessif d'affirmer qu'antérieure-[p. 13] ment au coup d'État la Grèce connaissait un régime de parfaite légalité. Aucune notion n'a été plus étrangère que celle de la primauté du droit à la pratique de la Droite sous ses diverses incarnations. Comme le montre bien le cas du gouvernement. Canellopoulos, constitué dans un mépris absolu des normes les plus élémentaires du système représentatif, cette attitude s'est maintenue jusqu'à la chute du régime parlementaire. Mais au moins avait-il été possible, au prix de luttes sévères, d'atténuer certains excès de la surveillance et de la répression policière, de procéder, non sans risques d'ailleurs, à la dénonciation publique de ces abus, d'élargir progressivement la sphère des libertés civiques.

Le dispositif d'oppression des colonels - dispositif dont certains éléments bouffons, voire loufoques, ne doivent pas masquer la nature tragique - demeure encore à l'abri de telles failles malgré tant de belles proclamations de soi-disant clémence à destination de l'opinion internationale. Désormais le fameux certificat de civisme n'est plus exigé en principe que pour les candidats à un poste relevant de la défense ou de la sécurité nationale mais tous ceux qui postulent un emploi gouvernemental au sens large du terme (administrations centrales et locales, organismes publics et parapublics) doivent remplir un questionnaire en dix points dont le cinquième concerne l'accomplissement éventuel d'actes « subversifs » par des membres de leur entourage familial immédiat. Si, par malheur pour lui, le postulant compte parmi ses parents un frère ou un oncle qui, avant le putsch, a participé à une marche de la paix, il est tenu de mentionner le point en indiquant son approbation ou sa désapprobation. Ce système qui résulte d'une décision prise au mois de janvier 1972 en dit long sur la manière dont les putschistes s'y prennent pour conserver l'arme de la peur en cette phase dite de « libéralisation ». 
Bien entendu ce comportement n'a rien de fortuit. L'on ne saurait attendre de conduite différente d'hommes [p. 14] n'ayant accédé au pouvoir et ne s'y étant maintenus que par le seul levier de la force et de la violence, d'hommes n'ayant pas osé, en cinq années de gouvernement dictatorial, organiser même un simulacre d'élection ouverte (le projet de constituer un parti, prêté à plusieurs reprises aux putschistes ne s'étant pas matérialisé). Le 21 avril 1967, la Grèce est tombée aux mains d'une bande de militaires de second plan dont le comportement s'explique beaucoup mieux par référence aux méthodes habituelles des gangs qu'aux schémas ordinaires du droit constitutionnel. Comme des poignées de gangsters ont pu régner sur de grandes villes en terrorisant la masse de la population et en s'assurant la complicité d'autorités officielles, ainsi les putschistes grecs ont-ils pu se constituer et conserver jusqu'à présent, grâce à de multiples complicités, nationales mais aussi étrangères, un domaine de chasse, le territoire de l'État grec, où il leur est possible d'assouvir leur soif inextinguible de pouvoir et des avantages matériels qui lui sont liés. Et les notions de « règlement de compte » ou d'une " solidarité de malfaiteurs » conviennent mieux que celles de responsabilité ou de cohésion ministérielle, et celle aussi d'honneur militaire, pour caractériser les rapports entre les factieux, pour saisir les traits essentiels de leur démarche.

Il serait certes facile d'exprimer les mêmes idées sous une forme plus savante, plus conforme au langage universitaire traditionnel. Ce faisant l'on aboutirait en définitive à rendre service aux hommes du putsch qui, en plus de dominer et d'exploiter la Grèce, entendent bénéficier du respect normalement accordé aux gouvernants d'un pays, jouir de tous les attributs habituels du pouvoir usurpé. Le souci d'adopter une forme correcte ne doit pas aller jusqu'au point où il aurait pour résultat de masquer la réalité de la situation. En traitant la Junte comme un gouvernement ordinaire parmi les autres on se fait, de manière consciente ou inconsciente, le complice de la dictature, on trahit le peuple grec étouffé par une machine armée forte de son appartenance à l'OTAN et de l'aide [p. 15] militaire américaine. Il y a peut être des putschistes, très rares à notre connaissance, qui n'ont pas profité de leur accès au pouvoir pour s'assurer des avantages indus, combler leur famille de bénéfices matériels ou encore donner libre cours à des instincts sadiques. Cependant l'on ne peut mieux qualifier les auteurs et profiteurs du coup d'État en tant que groupe que par application de la notion de gangstérisme politique : ayant brutalement conquis un pouvoir à l'exercice duquel rien, si ce n'est une propension paranoïaque, ne les destinait, ils ne sauraient en réalité avoir d'autre objectif, d'autre critère d'action que de prolonger autant que possible la durée du festin. Sur cette base, la peur que les putschistes s'efforcent d'inculquer à leurs adversaires n'a d'égale que leur propre peur (peur physique pour commencer: voir l'ampleur de la protection policière dont s'entoure Papadopoulos).

Une fois au pouvoir, et après s'être taillé dans la vie publique du pays des fiefs correspondant à leur ambition ou s'être mis en possession des instruments d'une ascension ultérieure, les colonels ont dû assurer le fonctionnement de l'État et la marche de l'économie dont ils venaient d'usurper le contrôle. Que ce soit par inclination idéologique, par souci de s'assurer la complicité toujours coûteuse des 
détenteurs du pouvoir économique et financier ou, tout simplement, par complète ignorance des problèmes fondamentaux de la société grecque, les putschistes se sont bornés à suivre les politiques imposées jusque là au pays par les couches dominantes et leur protecteur américain - continuité à peine altérée par l'extrême confusion introduite dans les services administratifs sous prétexte de révolution ou régénération nationale.

Les hommes de la Junte, auxquels certains commentateurs prêtaient au début, par analogie abusive, des desseins d'inspiration nassérienne, se sont limités de la sorte au rôle de garant des mécanismes et des relations du capitalisme, à une activité de protection de l'exploita-[p. 16] tion impérialiste sous toutes ses formes. Et comme les hommes actuellement au service de l'État grec, même s'ils manquent de courage ou de pudeur à l'égard des oppresseurs de leur pays, ne sont pas tous des profiteurs ou des ignorants, il est arrivé et il arrive encore que la dictature adopte sur leur suggestion des mesures conformes à l'avantage collectif, tel du moins qu'on peut le concevoir dans une optique capitaliste.

Ces influences technocratiques ne signifient certes pas que les technocrates ont remplacé les militaires à la tête du pays comme l'ont affirmé un peu vite certains journalistes étrangers au lendemain du dernier remaniement ministériel important (fin août 1971) mais il est bien possible que l'élimination des militaires de certains postes, quoique ne changeant rien à la nature profonde du régime, entraîne finalement diverses améliorations au titre de la gestion. Il pourrait en résulter une expansion des pourboires de la dictature et l'on se priverait en négligeant ce point d'une dimension indispensable à la compréhension totale du phénomène dans la mesure où ces pourboires valent aux putschistes en différents milieux plus de sympathies que leurs adversaires grecs ne l'admettent habituellement. En tout cas, les résultats ainsi acquis n'ont pas été suffisants pour que les factieux envisagent, à l'exception de la farce du référendum constitutionnel, de donner à leur régime un autre fondement que celui du hold-up initial.

Reconnaissons d'ailleurs que Papadopoulos n'a jamais fait mystère de sa profonde méfiance à l'égard du processus électoral : c'est même l'un des rares points sur lesquels ses discours nébuleux sont relativement clairs. Ainsi dans le discours prononcé lors de l'inauguration de la $36^{\mathrm{e}}$ Foire internationale de Salonique (septembre 1971) a-t-il pris soin de rejeter l'idée que la démocratie s'exprime uniquement, selon ses propres termes, par le vote et la chasse aux voix. Le colonel n'a pas caché en cette occasion que le régime ne saurait prendre le risque d'é-[p. 18] lections précoces. Un peu plus tard il est vrai, au cours d'une interview donnée à un journaliste américain, le général Pattakos, toujours à l'aise dans les rôles de bouffon, a pris sur lui d'affirmer que l'absence d'élections correspond au souhait des Grecs - ces Grecs qui, d'après le général, sont plus heureux et plus satisfaits que les gens de n'importe quel autre pays.

Ce schéma d'interprétation, dont nous n'excluons pas divers traits individuels de putschistes (l'ambition illimitée de certains d'entre eux, le sadisme et la cruauté de plusieurs autres, la complète imbécilité de quelques-uns), ne constitue sans doute pas une clé d'explication absolument totale. Cependant il permet de comprendre d'un 
coup plusieurs points essentiels et en particulier le fait que le régime utilise, doit utiliser pour se maintenir au pouvoir, des moyens sans aucune commune mesure avec les activités entreprises en dehors de l'auto-protection du gang. En d'autres termes, il n'est aucun des résultats acquis par la dictature, en particulier dans l'ordre économique et social, qui n'aurait pu l'être par les procédés gouvernementaux habituels du système capitaliste sans qu'il y ait lieu d'avoir recours aux techniques du totalitarisme et de l'univers concentrationnaire. Ce point est essentiel pour comprendre les mobiles ainsi d'ailleurs que les limites de l'opposition de la Droite à la dictature militaire.

[p. 18, sans texte] 
[p. 19]

\section{II \\ PORTÉE DE L'AFFRONTEMENT ENTRE LA JUNTE ET LA DROITE}

$\underline{\text { Retour à la table des matières }}$

Dans la pratique politique d'avant le putsch la notion de Droite telle que représentée par l'ERE se distinguait de celle du Centre telle que personnifiée par l'EK. Mais cette différence tendait à dissimuler et en tout cas à minimiser l'existence au sein de l'Union du Centre d'une aile conservatrice, bien proche pour l'essentiel de la Droite proprement dite (à l'exclusion sans doute de la frange carrément fasciste de celle-ci). Les dangers résultant de cette situation pour l'unité de la formation centriste allaient se manifester rapidement avec la crise de juillet 1965. Si l'on fait abstraction des séquelles des luttes politiques anciennes ainsi que des considérations purement électorales, il est bien difficile de relever des divergences significatives entre les positions d'un Canellopoulos et celles d'un Mavros : à l'égard des grands problèmes et des options essentielles de la société grecque, les facteurs de convergence semblent bien plus forts que les mobiles de séparation. Dans ces conditions, nous ne croyons pas faire violence à la réalité en rangeant ici sous l'étiquette de «Droite " non seulement le personnel de [p. 20] l'ERE mais aussi les éléments conservateurs de l'EK, cette entorse au vocabulaire partisan habituel étant largement compensée par une plus exacte appréciation du rapport des forces vis-à-vis de la dictature.

Très rapidement en tout cas, plusieurs hommes politiques d'idéologie conservatrice se sont rendu compte de l'énorme disproportion entre les politiques suivies et les moyens utilisés par les putschistes. Ils ont vite compris que le totalitarisme des colonels n'était pas commandé par les exigences de la défense capitaliste mais par la volonté d'un gang d'exploiter au maximum le pouvoir brutalement conquis. La conséquence logique de cette perception fut d'affirmer aussitôt l'aptitude des dirigeants conservateurs à assurer la protection du système social sans qu'il soit nécessaire d'utiliser à cet effet un appareil dictatorial. On doit reconnaître au surplus que chez plusieurs de ces leaders les attaques contre la Junte exprimaient une répugnance profonde à l'égard des méthodes de la tyrannie militaire. 
Ces mêmes milieux certes n'avaient pas toujours témoigné d'une confiance égale dans le fonctionnement des mécanismes constitutionnels ainsi que le montre leur participation active au coup de force royal de juillet 1965, lequel faisait fi ouvertement des normes fondamentales du parlementarisme bourgeois. Mais les circonstances même du putsch - en particulier la facilité avec laquelle une poignée de conspirateurs n'ayant derrière eux qu'un segment des forces armées purent neutraliser en quelques heures la plus large part des forces dirigeantes de la Gauche et du Centre Gauche dans le pays - soulignaient à elles seules la futilité des petites peurs des possédants.

Au titre du putsch d'avril 1967 en tout cas, le leader de l'ERE, P. Canellopoulos a contesté formellement que l'intervention des militaires ait été justifiée par l'éventualité d'un soulèvement communiste. Et la Commission Européenne des Droits de l'Homme lui a donné raison en se refusant à admettre qu'il existait en date du 21 avril 1967 [p. 21] « un danger public menaçant la vie de la nation grecque » (en particulier pas de menace d'un coup d'État communiste). On peut certes regretter que la Commission n'ait pas jugé utile de préciser qu'à l'époque le seul risque de subversion du régime représentatif tenait à l'éventualité d'un coup militaire sous une forme ou l'autre. Nous ne pouvons d'ailleurs que spéculer sur l'attitude qu'aurait prise la Droite à l'égard d'un coup réalisé par les généraux sous l'égide du Souverain intervention dont plusieurs témoignages autorisés (spécialement ceux de Zoïtakis et de Spandidakis) ont montré que les intéressés en avaient étudié le principe et, peutêtre, en avaient décidé la réalisation avant d'être pris de court par les colonels. Depuis lors, plusieurs des dirigeants de la Droite ont souligné publiquement à maintes reprises la nécessité d'en terminer avec la dictature. L'un des aspects essentiels de cette argumentation, spécialement destinée à calmer les appréhensions de Washington, est la possibilité d'assurer la succession des putschistes sans que le pays glisse vers ce que les conservateurs appellent le chaos, c'est-à-dire en réalité vers des changements socio-politique substantiels. En d'autres termes, selon les modérés, la dictature est inutile puisque l'ancienne classe politique se trouve en mesure, si on lui donne la possibilité de ressaisir le pouvoir, de maîtriser la situation sans recours aux solutions de force. Cette dictature est au surplus dangereuse pour l'ordre établi dans la mesure où ses cruels excès et son attirail idéologique grotesque ne peuvent qu'encourager le besoin de transformations profondes, voire même à la longue susciter de nouveaux risques d'affrontement sanglant. D'un autre côté, toujours selon cette thèse, les militaires compromettent par leur incompétence et leur ignorance les institutions et mécanismes de l'ordre capitaliste qu'ils se targuent de protéger (affirmation incessante, selon l'évangile des putschistes, du caractère " occidental » de la Grèce).

[p. 22] Ces attaques, quoique reposant parfois sur des données trop unilatérales ne doivent pas être négligées car les défauts et les risques qu'elles signalent correspondent bien à la réalité. L'état de la balance des paiements dont l'équilibre n'est assuré qu'au prix d'un endettement étranger croissant constitue à juste titre la cible de choix de ces dénonciations. Les dirigeants actuels de l'économie contestent la portée de ces critiques. Ainsi dans son rapport pour l'année 1970 le gouverneur de la Banque 
de Grèce, D. Galanis, affirme-t-il que pour les économies semblables à l'économie grecque il ne faut pas confondre l'existence de déficits croissants et d'un endettement étranger en expansion avec les symptômes du déséquilibre externe affectant des pays développés: selon lui, sous réserve que certaines conditions soient remplies, ces déficits et ces emprunts sont normaux et constituent des facteurs essentiels de croissance dans toute économie en voie de développement.

Il y aurait certes beaucoup à dire sur les considérations optimistes (ainsi mention des effets catastrophiques que pourrait avoir pour l'économie grecque la survenance de graves difficultés sur le marché financier international). Toutefois les attaques de la Droite seraient plus convaincantes si les colonels ne s'étaient bornés à suivre, plus ou moins adroitement, la route déjà fortement tracée par la Droite et qui est effectivement, ce que la Droite n'admet pas, celle de la servitude. Si l'économie nationale connaissait un jour les difficultés majeures et irrémédiables que lui prédisent certains dirigeants d'avant le putsch, il serait certes bien difficile d'exonérer ces derniers d'une grave responsabilité dans la débâcle. Une fois de plus, nous sommes ramenés à la constatation qu'il n'était vraiment pas nécessaire d'ouvrir à nouveau les camps de concentrations pour continuer des politiques de facilité et de désordre aboutissant à placer le pays sous la tutelle économique et financière de l'étranger.

Autre facteur d'agacement de la Droite, du moins de [p. 23] ses couches sociales les plus élevées (les soi-disant "grandes familles athéniennes ») : le fait que le pouvoir suprême soit tombé aux mains de parvenus dont l'élocution et les manières laissent apparemment beaucoup à désirer selon les standards du meilleur monde. C'est un fait que les putschistes viennent pour la plupart de familles très modestes et ne s'en cachent d'ailleurs pas mais s'étant assurés l'ascension politique ils veulent aussi conquérir l'ascension sociale d'où chez eux, ainsi que chez leurs femmes, les inévitables maladresses qui accompagnent le souci de métamorphose (nous sommes désormais bien loin du souci de pureté affiché à l'origine). La bourgeoisie athénienne se rit de ces faux pas et accepte difficilement que des gens d'aussi basse extraction à ses yeux détiennent les fonctions supérieures même si, comme classe, elle n'a pas à se plaindre de leurs activités. Une certaine fronde qui, toutes proportions gardées, rappelle les bouderies de l'aristocratie romaine vis-à-vis de Mussolini, n'a pas d'autre origine. En somme les valets ont pris la place des maîtres et, ayant pris goût au pouvoir, refusent de l'abandonner au profil de ceux qui s'en tiennent pour les détenteurs naturels.

Sans attacher à ces petites vanités plus d'importance socio-politique qu'elles n'en valent, c'est là sans doute un facteur de l'opposition qui se manifeste dans les salons athéniens à l'encontre des putschistes. Tout changerait bien sûr si la moindre menace contre les privilèges bourgeois se profitait à l'horizon: les grandes familles qui les brocardent aujourd'hui seraient alors bien aise de compter sur les «sauveurs de la patrie ».

Les milieux conservateurs ne sauraient critiquer très sérieusement les politiques de la Junte puisque, à quelques détails ou erreurs de manœuvre près, ce sont leurs 
propres objectifs que celle-ci entend réaliser. On s'enrichit sous la dictature comme on le faisait au temps de la démocratie bourgeoise. Caramanlis ou Papadopoulos, ce fut sous le gouvernement de l'un et c'est sous la dic-[p. 24] tature de l'autre le même culte du veau d'or. Les attaques contre la Junte portent donc essentiellement sur les conditions de gestion de l'ordre capitaliste et sur le contenu des méthodes de gouvernement dont le seul fondement a été et reste d'assurer le maintien au pouvoir, pour une durée indéfinie, des putschistes.

La Junte n'ayant rien changé aux assises de l'ordre social et aux mobiles de l'activité économique, la concentration des attaques sur les fautes de gestion commises par le régime et sur la nature dictatoriale des moyens utilisés revient simplement à préconiser la restauration du système politique antérieur au putsch avec, naturellement, retour sur le trône de Constantin, premier serviteur, quoique de manière particulièrement obtuse, du capital monopoliste. Que telle soit en définitive l'ambition de la Droite et plus largement du monde conservateur, le point ne saurait causer la moindre surprise. En bien pesant les mots, et sans la moindre crainte de commettre une injustice, il s'agit là de querelles de famille. Ces querelles peuvent certes être très violentes (voir les disputes sur les héritages) et il peut même arriver qu'elles soient sanglantes : m'ais, en définitive, elles ne suffisent pas pour bouleverser le fonctionnement social. La dictature n'a rien changé et sauf changement imprévisible ne changera rien au système de domination et d'exploitation du peuple grec par ce qui tient lieu à la Grèce de classe bourgeoise et par le capital étranger. Les luttes entre la Droite (Caramanlis en tête et Mavros y compris) et les putschistes se situent à l'intérieur de ce système : il s'agit seulement de savoir qui a le plus de titres pour assurer la perpétuation du désordre établi.

Il y a un terrain sur lequel l'identité des vues entre les conservateurs et les militaires est apparemment complète : celui de l'appartenance de la Grèce au système militaire de l'OTAN. Durant sa longue occupation du pouvoir, l'ERE s'en est tenue au respect intégral de la lettre et de l'esprit de l'Alliance - politique aboutissant en réa-[p. 25] lité à placer l'armée grecque et le système national de défense sous la stricte tutelle des États-Unis. Des leaders de la Droite ont même présenté l'acceptation des accords de Zurich comme l'expression d'une volonté de sauvegarder au maximum les relations atlantiques. La Droite a maintenu cette attitude dans l'opposition sous le gouvernement de l'Union du Centre (rappel de la tempête de protestations suscitée par le projet de voyage à Moscou de G. Papandréou ; rappel aussi du procès d'intention livré à A. Papandréou au sujet d'un éventuel relâchement de l'alliance). Or c'est là une position dont les putschistes n'ont jamais dévié depuis leur saisie du pouvoir : que ce soit en paroles ou en actes, la Junte s'en est toujours tenu au respect le plus minutieux, le plus complet des allégations atlantiques. La part des dépenses militaires dans le produit national et dans le budget témoigne avec éloquence de cette inaltérable fidélité.

Assez rapidement toutefois la Droite forte de son indéfectible attachement à l'Alliance, s'est efforcée de dissocier l'OTAN de la dictature et d'obtenir l'appui de la première pour jeter bas la seconde. Idée de base de cette manœuvre : la participation 
pleine et entière des putschistes au système otanique leur assure une particulière respectabilité dans le pays et contribue donc à renforcer leur position.

Si l'armée grecque avait le sentiment que la prolongation de la dictature risque de compromettre la solidité et la qualité des liens atlantiques, alors elle s'organiserait sans tarder pour chasser les colonels du pouvoir - prédiction qui d'ailleurs néglige l'importance du niveau des soldes et autres avantages matériels dans les comportements militaires. De toute manière comment obtenir la condamnation qui, selon cette interprétation, frapperait de manière décisive la dictature Papadopoulos ?

Une première ligne d'attaque pouvait être de souligner la contradiction entre les principes dont se réclame l'alliance (« les principes de la démocratie, les libertés indi[p. 26] viduelles et les principes du droit «selon le préambule du Traité ») et le maintien en son sein d'un régime fondé sur la négation même de ses principes. Mais, sauf dans une vue exagérément naïve du système atlantique, c'était et c'est toujours une argumentation de bien faible portée. Le Portugal appartient depuis le début à l'OTAN, la valeur stratégique des Açores compensant la rigueur de la police salazarienne. L'Espagne lui est étroitement associée dans le cadre de son alliance avec les États-Unis : or la somme d'horreurs et de massacres commise par les hommes du franquisme, même après la fin de la guerre civile, dépasse incommensurablement la somme des persécutions accomplies par les colonels grecs. Il y a aussi le cas de la Turquie qui ne constitue pas précisément un modèle de démocratie représentative : sans doute les militaires turcs ont-ils manœuvré de façon plus intelligente et plus souple que les putschistes helléniques mais les événements d'avril 1971 ont montré, sans aucune ambiguiité, que l'armée turque entend se réserver le contrôle supérieur de la politique nationale. Et dans ce pays la répression de l'opposition, quoique plus sélective qu'en Grèce, n'en est probablement pas moins efficace. Il est vraiment peu réaliste, et vu la présence de plusieurs dictatures dans le système il est même indécent, de s'adresser à l'OTAN pour en obtenir le respect par ses membres de la primauté du droit, même en termes de simple légalité bourgeoise.

Les adversaires de la Junte ont utilisé, il est vrai, une seconde ligne d'attaque consistant dans l'affirmation que la présence de la Junte tend à affaiblir considérablement la valeur de la Grèce comme membre de l'alliance, qu'elle a pour résultat d'en faire un maillon particulièrement faible du dispositif militaire allié. Cette affirmation s'appuie avant tout sur les dommages causés au potentiel de combat de l'armée grecque par les épurations massives d'éléments jugés hostiles ou peu loyaux à l'égard de la Junte, le renvoi des chefs les plus compétents et leur [p. 27] remplacement par des chefs de qualité médiocre, le niveau élevé de favoritisme et les mutations incessantes, la surveillance exercée sur les officiers supérieurs par certains de leurs subordonnés (les officiers « révolutionnaires »)... Ainsi, affirme-t-on dans les milieux hostiles à la Junte, une armée moderne a-t-elle été ravalée au niveau d'une force de répression dont la valeur combative n'excède pas celle des armées de l'époque des guerres balkaniques. Même si les colonels putschistes se prétendent les plus fidèles défenseurs de l'alliance, le plus simple souci d'efficacité militaire commande leur éloignement du pouvoir dans les délais les plus rapides. 
Cette argumentation a été utilisée à maintes reprises, en particulier au lendemain de la vaste épuration des cadres qui a suivi l'équipée royale du 13 décembre 1967. Ceux qui s'en servent ne cessent de dire que la présence de la dictature nuit au rendement militaire de l'alliance : il leur paraît difficile de croire que malgré les efforts accomplis par le général Anghelis durant ces dernières années la valeur de l'armée grecque comme instrument d'intervention extérieure n'ait pas souffert des innombrables purges et des graves tensions liées de façon directe aux luttes pour le contrôle et la conquête du pouvoir suprême (et, à cet égard, d'ailleurs c'est du soidisant complot de l'Aspida et non simplement du putsch qu'il faudrait partir pour évaluer les dommages). Il reste cependant que les dirigeants militaires de l'alliance, particulièrement les dirigeants américains, n'ont cessé d'apporter aux colonels des témoignages publics d'estime - lesquels, en certaines circonstances délicates pour la Junte, ont pu être interprétés à bon droit comme des marques sans équivoque de soutien. Aujourd'hui c'est en Grèce, et non en Turquie, que la marine américaine a décidé de créer un nouveau port d'attache (HOMEPORT) pour la $6^{\mathrm{e}}$ flotte : il s'agit de construire des installations capables d'abriter une partie de cette flotte ainsi que des habitations pour loger quelques milliers de membres du [p. 28] personnel naval et leur famille. Le New York Times a qualifié ce projet avec sagacité de «bonanza for the colonels » et de toute manière il reste significatif qu'il ait été conçu en dépit des attaques contre la continuation de l'aide militaire à la Junte.

Il semble bien d'ailleurs que pour les militaires de l'OTAN le point le plus faible de l'alliance ne soit pas le dispositif sud-est auquel appartient la Grèce mais bien plutôt le secteur Nord qui couvre la Norvège, le Danemark et l'État ouest-allemand du Schleswig-Holstein. C'est du moins ce que l'on peut déduire de récentes déclarations du général Sir Walter Walker commandant de ce secteur. Le général attribue cette situation à des déficiences sérieuses dans l'ordre des effectifs et de l'armement déficiences imputables aux politiques suivies par les gouvernements danois et norvégiens (maintien des effectifs très au-dessous des niveaux prévus, refus d'autoriser le stationnement des troupes alliées sur le territoire, refus d'accepter le dépôt d'armes nucléaires et de forces nucléaires...). Sans doute ces gouvernements, qui comptent parmi les adversaires les plus résolus de la Junte, ont-ils de très bonnes raisons d'agir de la sorte mais l'on ne saurait demander aux militaires de l'OTAN de préférer ce comportement à celui des putschistes, si avides d'aide militaire et si compréhensifs à l'égard des demandes de l'OTAN.

Une autre manière d'exprimer les torts causés à l'alliance atlantique par les putschistes est de souligner la montée en Grèce d'une attitude d'antiaméricanisme, y compris dans les milieux et les clientèles de la Droite, pourtant si favorables jusque là au développement et à la consolidation des liens avec les États-Unis, si amicaux en toutes occasions à l'égard des Américains. L'OTAN se confondant en Grèce avec les États-Unis, l'expansion d'une telle attitude ne peut que porter préjudice à l'alliance elle-même. Selon cette interprétation, le surgissement en Grèce d'un fort sentiment d'anti-américanisme tient à la [p. 29] conviction largement répandue que les ÉtatsUnis ont eu une responsabilité décisive dans l'arrivée et le maintien au pouvoir des putschistes : si Washington l'avait vraiment souhaité la dictature militaire aurait été 
abattue depuis fort longtemps. En s'étendant et en se généralisant, poursuivent les exposants de cette thèse, cette attitude ne peut que miner la confiance du peuple grec dans la valeur de l'alliance atlantique et le rendre plus réceptif aux suggestions d'un changement dans les relations internationales du pays.

Les renseignements disponibles ne permettent pas de vérifier l'ampleur de cette poussée d'antiaméricanisme, particulièrement dans les troupes électorales habituelles de la Droite. Si l'on s'en tient aux déclarations publiques faites par des dirigeants ou des personnalités de marque de ce secteur, il est hors de doute que plusieurs s'expriment avec beaucoup d'amertume sur le comportement de Washington et de l'OTAN à l'égard des putschistes. On entend ainsi d'anciens défenseurs inconditionnels de l'alliance formuler aujourd'hui des doutes et des réserves à l'encontre de la politique américaine - propension que ne pouvait manquer d'intensifier la décision annoncée par les États-Unis en décembre 1970 de reprendre les livraisons d'armes lourdes. Ces prises de position défavorables à l'Amérique correspondent peut être à un essai tactique de persuasion mais l'on ne saurait exclure qu'elles portent témoignage d'un désenchantement plus profond. Les dangers signalés par les défenseurs de la Junte qui se situent dans une perspective atlantique ne sont donc pas imaginaires du point de vue américain mais il est douteux que de telles considérations puissent modifier sensiblement la position des dirigeants militaires de l'OTAN lesquels préfèrent sans doute cet antiaméricanisme diffus à la présence d'un parti communiste opérant dans la légalité.

Les putschistes ont en effet un autre mérite aux yeux des militaires de l'OTAN : celui d'avoir entrepris et de [p. 30] poursuivre sans relâche l'annihilation systématique du mouvement communiste grec ainsi que des différents groupes de sympathisants. C'est là certes un front sur lequel la Droite a combattu avec beaucoup d'énergie et sans trop tenir compte des droits de l'homme : en cas de retour de celle-ci au pouvoir après les colonels, l'on peut penser qu'elle s'efforcerait de livrer le même combat avec toutes les armes disponibles. Mais les factieux sont en mesure d'asséner à l'opposition de gauche des coups encore plus rudes et les militaires ont volontiers propension à choisir la manière la plus forte pour réduire à merci ceux qu'ils tiennent pour dangereux à la sécurité nationale. Sur ces bases il est normal que les dirigeants de l'OTAN préfèrent la Junte à la Droite pour assurer la répression anticommuniste et cela en dépit des références acquises par la seconde en ce domaine depuis la fin de la guerre civile.

Jusqu'à maintenant, les adversaires de la Junte ne sont pas parvenus à retourner l'OTAN en leur faveur. Aussi plusieurs d'entre eux témoignent-ils de mauvaise humeur ou d'inquiétude, l'une n'excluant pas l'autre, à l'égard des positions de l'alliance vis-à-vis des putschistes. Selon eux le maintien intégral des relations militaires et a fortiori leur développement ne peuvent que consolider la position politique du régime tant sur le plan extérieur qu'intérieur... Et les factieux bénéficient de tels avantages, que les pays impliqués, au premier rang desquels les États-Unis le veuillent ou non... Le souci de garder les putschistes comme alliés militaires ne peut que conduire à les ménager sur d'autres plans (politique), voire à les aider (crédits, 
investissements). En d'autres termes, il n'est pas possible de dissocier les différents domaines : le rejet doit être total pour avoir la moindre efficacité.

Telle n'est pas la politique préconisée par C.L. Sulzberger vis-à-vis du gouvernement militaire. D'après lui la politique américaine en ce domaine (A SANE U.S. [p. 31] POLICY FOR GREECE) devrait s'inspirer des deux principes suivants : faire pression sur le gouvernement d'Athènes par tous les moyens possibles (IN EVERY POSSIBLE WAY) pour obtenir la restauration de la liberté et également entretenir des relations étendues avec l'opposition ; reconnaître à la Grèce comme une alliée dans l'OTAN le droit à un armement moderne. Un tank M-48 n'est pas plus utile contre des foules hostiles qu'un tank M-47 mais le premier a certainement une utilité plus grande que le second contre un adversaire étranger potentiel. Les ÉtatsUnis ont besoin des bases navales et aériennes de la Grèce pour tenir leurs engagements dans une région du monde qui leur est désormais largement hostile. Encore que Sulzberger ne le dise pas expressément, on peut déduire de ces deux principes que si les pressions pour la liberté connaissent l'échec (la dictature maintenant, voire élargissant et perfectionnant son appareil répressif), l'aide militaire ne doit pas être interrompue pour autant. En somme l'intérêt des États-Unis exige que ceux-ci tiennent les deux plans d'intervention soigneusement séparés - position qui dans l'état actuel revient à n'attribuer qu'un rôle secondaire à l'objectif du rétablissement de la liberté en Grèce.

Depuis le putsch, la collaboration militaire des Américains avec la dictature a été bien plus large et bien plus intense que la pression des premiers sur la seconde pour le passage à un régime moins abject. Sur le premier plan, il y a eu des actes nombreux ostensibles et lourds de signification (participation combinée à des manœuvres d'inspiration agressive, par exemple) tandis que sur le second les autorités de Washington, particulièrement celles de la branche exécutive, se sont bornées au maximum à des observations et suggestions discrètes. Ceux qui tiennent l'OTAN pour une alliance de peuples épris de paix et de liberté peuvent bien s'étonner d'un tel décalage et le déplorer mais cette contradiction entre les principes et les faits ne saurait surprendre ceux qui considè-[p. 32] rent cet organisme comme un instrument de force au service d'un impérialisme agressif. En s'adressant à l'OTAN contre les putschistes on se range inévitablement dans le camp de ceux pour lesquels la restauration de la liberté consiste seulement à troquer les militaires factieux contre les conservateurs civils de l'ordre établi. Quand la Droite agit de la sorte, elle est simplement logique avec elle-même et l'on ne saurait lui reprocher de manquer de cohérence ou de persévérance. Il est plus étonnant que des esprits d'intention réformiste ou progressiste cèdent à une telle mystification. Sont-ils nombreux ? 
[p. 33]

\section{III \\ À PROPOS DES TACTIQUES UNITAIRES}

$\underline{\text { Retour à la table des matières }}$

Quoique les Américains aient considérablement aidé les putschistes de plusieurs manières, quoique leurs efforts pour le rétablissement du régime représentatif aient été et demeurent fort réservés, quoique certains de leurs services préfèrent sans doute à toute autre formule la continuation du système actuel, les autorités de Washington verraient probablement d'un bon œil le retour au pouvoir d'une équipe d'esprit conservateur - une équipe capable de leur assurer, dans un cadre de démocratie bourgeoise, le maintien des avantages acquis en Grèce avant et depuis le putsch. Les intérêts de la Droite et ceux des Américains sont intimement liés en dépit de l'amertume et du désenchantement qu'affiche actuellement la première à l'égard des seconds. En certaines circonstances, l'appui des États-Unis et de l'OTAN (c'est d'ailleurs pratiquement la même chose) pourrait faciliter considérablement la réinstallation de la Droite au pouvoir, que ce soit sous la direction de Caramanlis ou d'un leader un peu moins voyant, un peu moins enclin à l'autoritarisme.

[p. 34] La Droite dispose d'ailleurs d'autres atouts pour une éventuelle reconquête du pouvoir. L'Union du Centre a perdu le seul homme qui avait été capable de réaliser la coexistence en son sein d'ailes d'inspiration opposée (et ce n'est pas le pâle Mavros qui pourrait le remplacer dans cette tâche). La plupart des dirigeants de la Gauche ont été traités avec une sauvagerie inimaginable et plusieurs personnalités du courant centre-gauche ont fait aussi l'objet de dures persécutions. En revanche la Droite a conservé tous ses leaders (y compris Caramanlis à Paris) : tout en ayant à subir des vexations policières et des atteintes à leur liberté (assignation à résidence), ces dirigeants ont quand même joui d'une plus grande latitude de manœuvre que leurs homologues des autres partis. Ainsi ont-ils pu à diverses reprises faire des déclarations, spécialement à des journaux étrangers, qui leur ont permis de demeurer dans l'actualité. En plusieurs cas, les media bourgeois ont donné une grande publicité à leurs attaques et présenté sur eux des commentaires fort élogieux. Enfin la Droite a bénéficié des réflexes anticommuniste et antisoviétique qui caractérisent encore tant de secteurs de l'opinion dans les pays capitalistes : ainsi s'explique que des hommes 
politiques portant l'étiquette « socialiste » (au sens de l'internationale Socialiste il est vrai) aient pu se déclarer favorables au principe d'une « solution Caramanlis ».

Dans ces conditions, faut-il donc tenir pour inévitable, la perspective d'un remplacement des putschistes par une équipe qui, sous couleur d'union nationale, garantirait à la Droite une large part dans la restauration de la démocratie représentative ? Faut-il admettre que vu le rapport des forces, la présence des ÉtatsUnis, l'état de l'opinion nationale et étrangère, c'est là en réalité la seule solution concevable ? Sans pouvoir indiquer avec exactitude la proportion de ceux qui pensent de la sorte, il semble que l'éventualité d'une solution genre Caramanlis soit admise, de plus ou moins bon gré, par des gens qui ne se rat-[p. 35] tachent à la Droite ni par leur passé, ni par leurs conceptions actuelles.

C'est ainsi que des hommes se disant par ailleurs d'inspiration réformiste déclarent accepter d'avance la présence dans un gouvernement post-dictatorial d'hommes dont ils dénonçaient jadis, à juste titre, la complicité foncière avec le gouvernement parallèle. Et certains d'entre eux franchissent un pas de plus, et fort grave, sur la voie de la soumission aux objectifs de la Droite en acceptant de ne pas poser la question de la monarchie, en ne faisant pas du départ définitif de Constantin la condition préalable d'une quelconque collaboration en vue de chasser les colonels du pouvoir.

Les motifs invoqués pour justifier l'adoption d'attitudes modérées par les milieux d'intention réformiste, lesquels ne tombent d'ailleurs pas tous dans ce piège, sont bien connus pour avoir été invoqués souvent en des circonstances voisines. Il s'agit simplement, mutatis mutandis, de l'application au cas grec de ces vieilles formules d'unité nationale qui, en bien des cas, ont permis aux milieux de l'oligarchie bourgeoise de garder les leviers de commande au lendemain d'une période dictatoriale dont ils avaient pourtant tiré de substantiels avantages. C'est aussi bien entendu l'appel au réalisme qui, dans le cas présent, invoque le souci de ne pas jeter ou rejeter les milieux conservateurs dans les bras des colonels et souligne la nécessité de ne pas effaroucher Washington. C'est également l'argument classique de l'urgence morale de l'union nationale contre les tyrans - argument qui serait certes plus convaincant s'il n'impliquait en toute occasion l'alignement de la gauche sur la droite, jamais le mouvement inverse. C'est enfin l'appel au souci d'efficacité : divisés, nous périrons, tous unis, nous renverserons la dictature (affirmation qui paraît correspondre au bon sens et qui est pourtant assez naïve dans la mesure où elle escamote la véritable difficulté qui serait de transformer un mouvement latent d'opinion [p. 36] en une force active de rupture ou de destruction de la dictature et d'empêcher les oppositions de resurgir sous l'impact des exigences mêmes de cette transformation).

Certains de ces groupes, il est vrai, n'acceptent l'hypothèse d'un gouvernement conservateur pour liquider les séquelles de la dictature qu'à titre d'expédient tactique : ils croient, éventuellement de très bonne foi, en la possibilité d'une seconde phase durant laquelle pourrait être élargie et consolidée l'assise démocratique du pays grâce à un ensemble de réformes appropriées. C'est là une position qui pourrait valoir à ses tenants bien des déceptions et même de cruels déboires : elle peut conduire à négliger ou à gaspiller la période, éventuellement courte, durant laquelle des réformes 
profondes pourraient être faites. Dans une situation comme celle de la Grèce, où les forces conservatrices disposent de tellement d'atouts, il paraît bien difficile de croire que la vieille classe politique ne profiterait pas d'un retour initial au pouvoir pour bloquer la réalisation des liquidations indispensables et pour stopper de nouveau la modernisation démocratique des structures socio-politiques. Les hommes des groupes réformistes qui fournissent aujourd'hui leur caution aux projets et manigances des adeptes du conservatisme et autres partisans de la monarchie font simplement une politique de gribouille : c'est un retour à 1950 et non pas même à 1964 comme certains le souhaitent, qu'ils contribuent à préparer. Faute de réaliser d'emblée les changements correspondant à la soi-disant deuxième phase, leur arrivée peut se faire attendre longtemps.

$\mathrm{Au}$ total ces positions de compromis qui impliquent des concessions toujours unilatérales sont fort dangereuses pour l'avenir démocratique du pays. Les hommes d'esprit libéral qui les adoptent, parfois il est vrai avec beaucoup d'hésitations, tendent ainsi à affaiblir les positions revendiquant la possibilité pour la Grèce d'adopter des voies nouvelles au lieu d'en revenir simplement aux politiciens et aux pratiques de l'ère pré-dictatoriale. Ils [p. 37] risquent ainsi d'oublier que dans une Grèce libérée de la tyrannie la première tâche d'une équipe démocratique du gouvernement, la plus urgente, devrait être, en s'appuyant sur les masses, de mettre radicalement fin aux institutions et aux mœurs ayant permis la réalisation du putsch. Et, à cet égard, même si des hommes de tendances conservatrices ont pu faire des actions d'éclat contre les colonels, ces mérites personnels n'enlèvent rien aux responsabilités écrasantes de la Droite dans la corruption des institutions parlementaires, le blocage des aspirations à la modernité socio-politique. Les vieux politiciens ne doivent pas profiter des immenses sacrifices consentis par les authentiques résistants.

Ces observations visent notamment la personne du Souverain dont l'ambition, les conceptions rétrogrades, le manque absolu du rayonnement moral et l'ignorance (stupéfiante même pour un roi) ont largement contribué à déclencher les conditions objectives et le climat psychologique propices à la réalisation du putsch. De telles responsabilités ne sauraient être prescrites par l'exil (un exil au surplus financé par les colonels). Dès lors même si des officiers se qualifiant de royalistes font exploser quelques bombes, même si des milieux des forces armées devaient participer à la chute de la dictature au nom de leur serment de fidélité au Roi, il serait déraisonnable et, en tout cas, fort dangereux pour l'avenir de la démocratie en Grèce de tirer de ces activités une sorte de réhabilitation du monarque. Le silence prudent observé par Constantin, dont la seule pensée est de ne fermer aucune des avenues pouvant le conduire au retour sur le trône, devrait suffire pour imposer sa disqualification définitive sauf bien entendu dans une perspective de retour intégral à l'ordre ancien.

Il reste à évoquer la position des Communistes et des hommes qui, luttent à leurs côtés. C'est eux qui, dès les premières heures de la dictature, ont eu à subir les assauts les plus féroces de la répression militaire. Mais, [p. 38] précédemment, la Droite ne leur avait pas fait beaucoup de quartier et l'on peut raisonnablement douter que la présence de la dictature ait sensiblement modifié cette attitude. Pour les Communistes, selon toute vraisemblance la chute de la Junte signifiera seulement la 
nécessité de livrer une autre bataille contre le monde capitaliste ou plus exactement de continuer la lutte actuelle sous une autre forme - une forme moins sauvage et barbare sans doute mais finalement aussi dure. Les Conservateurs étant ce qu'ils sont en Grèce, il serait fort étonnant que les milieux bourgeois tiennent le moindre compte des sacrifices subis par les hommes de la Gauche. Tout ce que ceux-ci pourront obtenir - par exemple la légalisation du Parti communiste - le sera par la lutte. Tous les appels à l'unité nationale lancés de la Gauche ne changeront rien aux comportements de la Droite - laquelle ne manquera même pas d'utiliser en les grossissant et les dénaturant certains comportements actuels de la Russie et des pays de l'Est comme argument de propagande contre le socialisme.

Il est vrai que pour toute une série de raisons, historiques et actuelles, la Gauche grecque se trouve aujourd'hui en position de faiblesse. Mais ce n'est pas en prêchant l'unité de tous les Grecs que l'on parviendra à convertir cette situation en une position de force. On peut admettre sans pessimisme excessif que les États-Unis, l'OTAN et le capitalisme international feront tout en leur pouvoir pour que, conformément aux vœux de la Droite, le remplacement de la Junte s'effectue dans le respect le plus complet de l'ordre bourgeois, pour que le changement porte seulement sur le mode de défense du système établi, en bref pour que le pouvoir passe d'un type de Droite à un autre. Et la Gauche aura bien de la chance si les forces dominantes jugent expédient de ne pas pousser aux leviers de commande les hommes les plus réactionnaires, de ne pas écarter systématiquement des nouvelles équipes gouvernementales les partisans d'une ligne plus [p. 39] modérée, un peu plus centriste.

Ce sont ces perspectives qu'il faut dénoncer sans relâche plutôt que de s'attacher à la promotion d'une unité nationale mythique et fallacieuse. Même si les chances d'influer ainsi sur le destin national demeurent minces, au moins pour l'immédiat, c'est la carte que la Gauche doit jouer si elle entend ne pas dévier du rôle qui doit être le sien. La Gauche doit établir par tous les moyens en son pouvoir que le problème n'est pas seulement de chasser les colonels du pouvoir même si tel est pour le moment l'objectif numéro un mais qu'il convient aussi de barrer le retour au pouvoir, fut-ce pour une période dite de transition, des hommes qui ont rendu possible la dictature et qui pour la plupart préféreraient conserver la dictature si son renversement devait déboucher sur une authentique démocratie, sur le socialisme. De toute manière, la Gauche en préconisant l'unité nationale n'attendra rien de ses adversaires de la Droite sous l'une ou l'autre de ses incarnations, rien si ce n'est une accusation de duplicité ou de fourberie. Dès lors le principe de son combat ne peut être que la continuation de la lutte contre les putschistes et de manière implacable mais aussi, et sans jamais faire de concession, le maintien de la lutte contre le capitalisme et l'impérialisme sans la destruction radicale desquels le recommencement de la dictature, d'un type ou d'un autre, ne sera jamais exclu.

Aujourd'hui le principal obstacle à une action de cet ordre réside dans la division de la Gauche. À cet égard la division du parti communiste et des organismes qui lui sont liés en deux segments hostiles constitue une réelle tragédie. C'est à la réunification des forces ainsi divisées que devraient être essentiellement consacrées les énergies unitaires. Cette séparation a d'autres causes que les divergences sur 
l'affaire tchèque mais celles-ci ont incontestablement tendu à élargir le fossé, à cristalliser les oppositions. Tout un courant qui fait siennes, sans broncher, les positions de la Droite et de la réaction [p. 40] mondiales soutient que l'Union soviétique s'est conduite en Tchécoslovaquie comme les États-Unis en Grèce, l'intervention de Prague correspondant en somme au putsch d'Athènes. Dans les deux cas, dit ce courant, il s'agissait pour les puissances intéressées de sauvegarder l'intégrité de zones d'influence, l'un comme l'autre des deux grands États se gardant bien, malgré beaucoup de protestations verbales, de gêner l'autre.

Sans entreprendre ici la discussion du dossier, disons trouver fâcheux que les dénonciateurs d'inspiration progressiste de l'action soviétique n'aient pas jugé opportun d'analyser en détails les manœuvres des forces impérialistes en Tchécoslovaquie (notamment celles des services de renseignements et des hommes d'affaires américains et ouest-allemands). Cet examen, comme celui de bien d'autres indices, leur aurait peut être permis d'apercevoir un autre principe de convergence entre les événements d'Athènes et de Prague, l'intervention agissante du capitalisme international visant dans le cas grec à éviter tout glissement du pays vers la contestation de l'impérialisme et dans le cas tchèque à réaliser, comme premier objectif, la dissociation du monde socialiste. Empêcher l'instauration du socialisme même à long terme, revenir sur l'instauration du socialisme par l'intensification des rapports économiques et culturel avec le monde capitaliste : ce sont là en réalité deux aspects complémentaires d'un objectif central, la lutte contre le socialisme et si l'on admet que tels sont les buts de la diplomatie impérialiste, l'intervention soviétique en Tchécoslovaquie prend une toute autre signification. 
[p. 41]

\section{IV \\ AU PEUPLE GREC DE CHOISIR SA DESTINÉE}

$\underline{\text { Retour à la table des matières }}$

Tous les raisonnements et anticipations que l'on vient de présenter ont habituellement un défaut grave qui est de ne pas tenir suffisamment compte des réactions possibles des masses, une fois celles-ci débarrassées du carcan dictatorial. Certains pensent ou espèrent que le soulagement procuré par la chute d'un gouvernement ignominieux sera assez profond pour que les Grecs acceptent sans opposition majeure, le retour sur la scène des hommes de l'ordre bourgeois, pourtant authentique fourriers de la dictature des putschistes - retour que les intéressés ne manqueraient certainement pas d'assortir de plus belles promesses de renouveau et de prospérité. Si tel devrait être le cas, ce serait pour la Droite, quelle que soit la forme sous laquelle on la présenterait au peuple, une victoire majeure.

Une telle perspective n'est pas invraisemblable mais on ne saurait la tenir pour nécessairement acquise. Les conditions dans lesquelles s'effectuera la libération du pays pèseront sans nul doute d'un grand poids dans la détermination des orientations futures de la politique grecque. [p. 42] De toute manière, ce n'est pas avec l'adoption de projets de compromis, lesquels entraînent inévitablement des attitudes de compromissions, que l'on galvanisera l'énergie populaire sans la mobilisation permanente de laquelle la réalisation d'un putsch militaire d'un type ou d'un autre restera toujours du domaine des possibilités. C'est là un mobile suffisant pour proposer au peuple grec des solutions de rechange claires et sans équivoque, des solutions qui, en plus d'un rejet absolu des techniques dictatoriales, comportent la fixation d'objectifs susceptibles d'assurer le changement national.

L'on ne saurait obtenir l'adhésion des masses aux exigences de cette transformation sans lui indiquer à l'avance les motifs, les étapes et l'aboutissement final de l'entreprise. Il est clair qu'une telle entreprise implique un rappel des faits antérieurs et un ensemble de jugements qui risquent de heurter les partisans de la tactique d'union nationale. 
Examinons, en particulier, la question de la monarchie. En considérant uniquement l'insignifiance de Constantin, on pourrait être tenté d'y voir seulement une question secondaire dont, de toute manière, la solution quelle qu'elle soit ne saurait avoir une influence décisive sur le destin national. Dans la mesure où la Grèce n'est pas le Danemark, ce serait là une erreur très grave. La monarchie en Grèce ne représente pas une institution purement symbolique ou décorative : elle correspond à toute une mentalité conservatrice, plus encore à toute une structure de relations (le « clan royal ») voué à la défense de l'ordre établi, à tout un système de pouvoir (lequel, actuellement en veilleuse, pourrait sans doute se reconstituer assez vite). L'abolition de la monarchie en Grèce ne résoudra pas par elle-même les difficultés de fond de la société grecque mais elle contribuera largement à déblayer le terrain, elle atténuera la résistance institutionnelle au changement.

[p. 43] On peut ne pas approuver toutes les thèses soutenues par André Papandréou depuis son départ de Grèce. Cependant on ne peut que lui donner raison quand il déclare que le Roi ne devra pas rentrer en Grèce après la chute de la Junte, sauf si le peuple en décidait autrement. Mais il y a le précédent du référendum du $1^{\mathrm{er}}$ septembre 1946 qui donne à réfléchir. Imaginons que le premier gouvernement de la Grèce libérée soit un gouvernement d'union nationale présidé par Caramanlis : on peut admettre à l'avance qu'une telle équipe ne manquerait pas d'agir, par tous les moyens en son pouvoir, pour obtenir un retour du roi. Or, en plus de tous les moyens habituels, ce gouvernement disposerait d'un réseau complet de media électroniques (selon les informations disponibles, le réseau de télévision en cours d'établissement doit être achevé assez rapidement d'où la possibilité prochaine pour les autorités gouvernementales d'atteindre par ce medium la Grèce toute entière). Il est donc dès maintenant indispensable de ne pas transiger dans la dénonciation du rôle néfaste joué par la monarchie grecque jusqu'au putsch de 1967 inclus. Le Roi n'est en aucune manière une victime de la Junte : il en a préparé directement l'avènement et il en a facilité considérablement l'établissement. C'est un point qu'il faut signaler sans cesse si l'on veut éviter qu'un référendum ultérieur sur la question royale soit un simple simulacre.

Cet indispensable effort de démystification - nécessaire pour que le peuple soit capable de se prononcer en connaissance de cause - implique le refus de passer l'éponge sur les luttes antérieures et sur les attitudes qui ont eu pour résultat les désastres de la dictature. Il implique aussi que l'on accepte que les Grecs, parce qu'ils ont des intérêts différents, professent des idéologies également différentes et que la lutte contre les colonels a des sens divers selon les positions de classe. D'aucuns ne manquerait pas de dire qu'une telle bataille - pourtant conforme à la plus stricte honnêteté politique - ne peut, [p. 44] en définitive, que profiter à la Junte. Répondons-leur simplement que le culte de l'union nationale à tout prix ne peut, en définitive, que favoriser la Droite sans qu'il soit démontré que la réalisation éventuelle d'un tel concours, nécessairement vague et superficiel, pourrait, comme tel, avancer d'un jour la liquidation des putschistes.

Si l'on va au fond des choses, la constitution et la sauvegarde de l'unité nationale jusqu'à la chute des colonels supposent un mode de libération dans lequel 
n'interviennent pas les forces populaires avec leurs objectifs de transformation et de libération propres, lesquels, effrayant aussitôt les classes possédantes (et, en Grèce, il en faut vraiment très peu pour effaroucher celles-ci) rompraient automatiquement l'unité. En revanche, il est vrai, l'unité est compatible avec d'autres modes de libération. Par exemple, le renversement des tyrans par une hypothétique intervention des États-Unis: dans ce cas, selon une vue assez naïve, l'unité pourrait même accélérer le processus de liquidation en incitant, voire en contraignant moralement, les Américains à agir. Ou encore la destruction de la Junte par intervention de militaires d'inspiration anti-dictatoriale (en somme une version améliorée du contrecoup de décembre 1967). Les dirigeants et porte-parole des milieux progressistes qui préconisent l'unité nationale ("des royalistes aux communistes ») ont-ils donc la conviction que le renversement des tyrans peut seulement venir de milieux extra populaires, sinon même, de manière plus ou moins franche anti-populaire ?

Jusqu'à maintenant, en tout cas, les espoirs d'un renversement de la dictature par action américaine ou contre-coup militaire (ou par une combinaison des deux) ne se sont pas matérialisées. D'un autre côté, il est vrai, les conditions d'une poussée populaire assez forte pour provoquer l'effondrement des tyrans ne semblent pas actuellement réunies. Et pourtant, chaque jour, tant sur le marché du travail en Grèce que sur la foire aux esclaves de l'émigration, des masses de travailleurs grecs sont [p. 45] aux prises avec les contradictions d'une croissance étroitement dépendante du capitalisme international. Si la dictature se prolonge, le gang des putschistes éprouvera, de par la structure même de son pouvoir, des difficultés grandissantes à administrer ces contradictions : des perspectives de lutte qui paraissent aujourd'hui quelque peu utopiques pourront devenir d'actualité. On s'apercevra alors que le combat des travailleurs contre la dictature diffère de celui des couches qui, tout en frondant le régime, en tirent de notables enrichissements.

La dictature actuelle est pour la plus large part le résultat de la stupidité politique et de l'aveuglement social de la classe dirigeante traditionnelle. Si celle-ci se révèle incapable de débarrasser le pays à ses conditions et par ses propres moyens de la tyrannie où elle l'a plongé, ce sont les couches populaires qui, en plus de l'exploitation capitaliste et de la persécution policière, auront à prendre ces risques et à assumer les coûts de la libération. Au moins que tous les efforts soient accomplis pour que les épreuves déjà subies et les sacrifices à venir n'aboutissent pas, comme ce fut déjà le cas en 1944, à la simple restauration de l'ordre ancien. 